\title{
Effects of partial closure and friction on a radial crack emanating from a circular hole
}

\author{
MARIA COMNINOU ${ }^{(1)}$ and F.-K. CHANG ${ }^{(2)}$ \\ (1) Department of Mechanical Engineering and Applied Mechanics, \\ University of Michigan, Ann Arbor, MI 48109, USA \\ (2) Department of Aeronautics and Astronautics, \\ Stanford University Stanford, CA 94305, USA
}

(Received July 21, 1984; in revised form November 15, 1984)

\begin{abstract}
A radial crack emanating from a circular hole in an infinite sheet under tension is considered. Depending on the crack length and location, the crack may be partially or completely open or closed. In case of closure, interfacial friction influences the solution.

Stress intensity factors for inclined tension or in-plane shear are computed and compared with those obtained when closure and friction are ignored.
\end{abstract}

\section{Introduction}

Radial cracks emanating from circular holes have been the subject of many publications. For a single radial crack, we mention the work of Bowie [1], Tweed and Rooke [2] and Hsu [3]. In addition, Atkinson [4] and Erdogan and Gupta [5] have considered the problem of an inclusion instead of a hole, the latter being obtained as a special case. In [3] the crack was disturbing either a locally tensile field and was, therefore, completely open, or a substantially compressive field and it should be partially closed. The analysis was, however, performed for an open crack in both cases, so that some negative stress intensity factors occurred in mode I.

The stress field around a circular hole in an infinite sheet in tension [6] shows that compressive circumferential stresses occur for angles $\theta<30^{\circ}$ and radial distances not exceeding $\sqrt{3 R}$, where $R$ is the radius of the hole, Fig. 1 (only the first quadrant is considered because of symmetry). A radial crack crossing this region may be expected to be partially closed. Along the contact, frictional forces are assumed to be transmitted according to the Coulomb law of friction. In general there will be regions of stick, slip and separation along the crack, and the extents of these regions must be determined. The effect of friction is also considered for shearing load at infinity, in which case the crack remains completely closed in the first quadrant.

The problem is formulated in terms of singular integral equations using the solution of a single dislocation and circular inclusion, Dundurs and Mura [7], as a Green's function. The same approach was used in [4] and [5]. A comparison is made with the stress intensity factors obtained in [3].

\section{Formulation}

Consider a crack of length $(c-R)$ emanating from a hole of radius $R$ in an infinitely extended solid. Uniform tension $T$ is applied at infinity at an angle $\theta$ from the crack axis, 


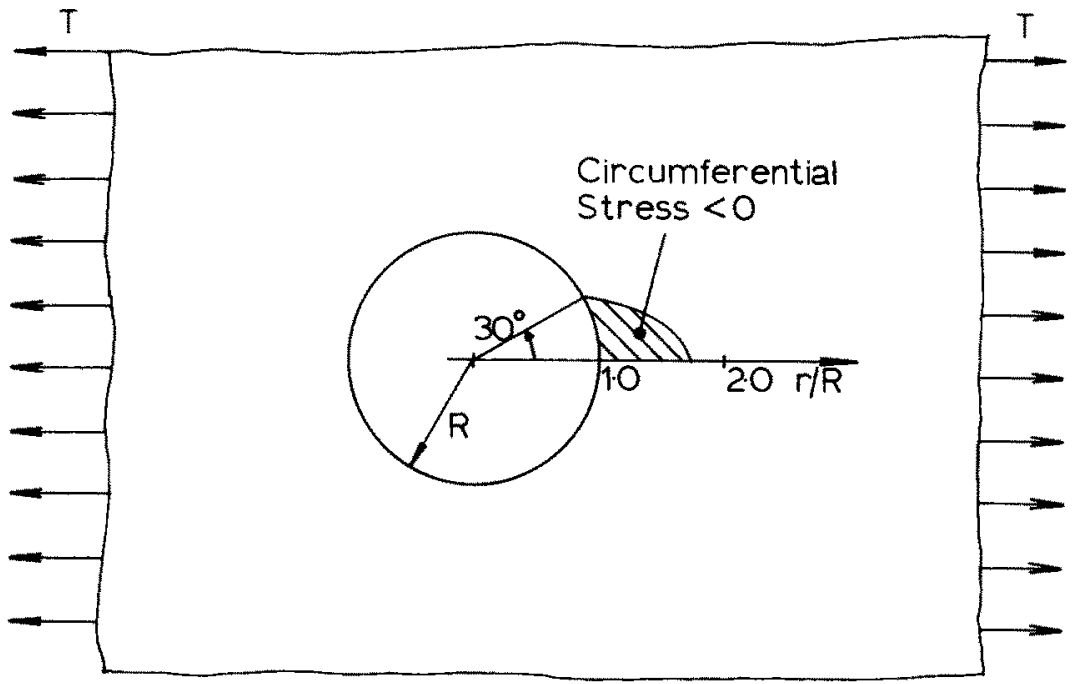

Figure 1. Region of compressive normal tractions along radial lines.

as shown in Fig. 2. Attention is restricted to values of $\theta$ and $c$ for which partial or complete closure occurs. Assuming that the crack faces are capable of transmitting frictional forces according to the Coulomb law there will be in general a zone of stick, $R<r<a$, and a zone of slip, $a<r<b$. The tractions along radial lines in the absence of the crack are [6]

$$
\begin{aligned}
& \sigma_{y y}(r, \theta)=\frac{1}{2} T\left[1+\frac{R^{2}}{r^{2}}-\left(1+3 \frac{R^{4}}{r^{4}}\right) \cos 2 \theta\right] . \\
& \sigma_{x y}(r, \theta)=\frac{1}{2} T\left[-1-\frac{2 R^{2}}{r^{2}}+3 \frac{R^{4}}{r^{4}}\right] \sin 2 \theta .
\end{aligned}
$$

The tractions due to a distribution of climb dislocations $B_{y}$ and a distribution of glide dislocations $B_{x}$ in a solid with a circular hole are on $y=0$ [7]

$$
\tau_{y y}(x)=\frac{2 \mu}{\pi(\kappa+1)} \int_{b}^{c} B_{y}(\xi) K_{y}(x, \xi) \mathrm{d} \xi
$$

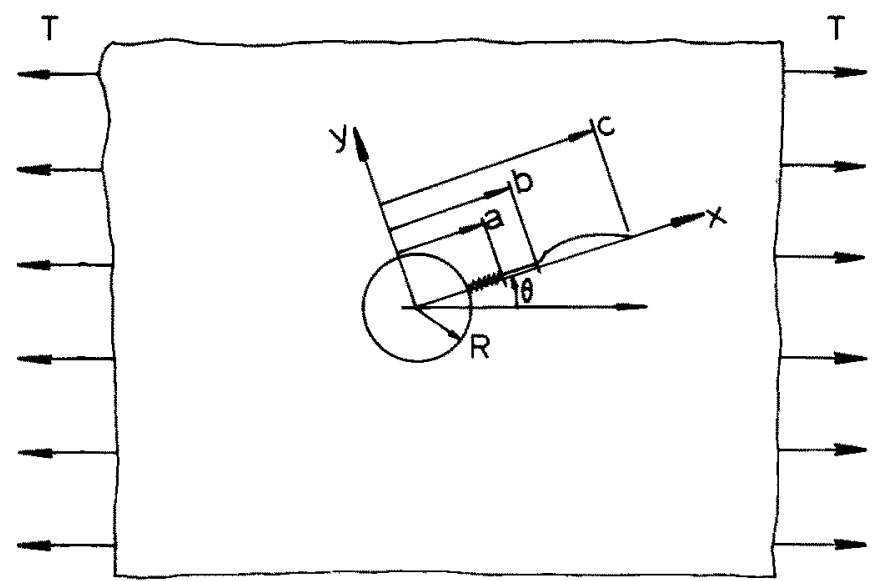

Figure 2. Geometry of the radial crack in an inclined tension field. 


$$
\begin{aligned}
& \tau_{x y}(x)=\frac{2 \mu}{\pi(\kappa+1)} \int_{a}^{c} B_{x}(\xi) K_{x}(x, \xi) \mathrm{d} \xi \\
& K_{y}(x, \xi)=\frac{1}{x-\xi}-\frac{\xi}{x \xi-R^{2}}-\frac{\left(\xi^{2}-R^{2}\right) R^{2}}{\xi\left(\xi x-R^{2}\right)^{2}}\left[\frac{\xi^{2}}{R^{2}}-\frac{\xi^{2}-R^{2}}{\xi x-R^{2}}\right]+\frac{\xi^{2}-R^{2}}{\xi x^{2}} \\
& K_{x}(x, \xi)=\frac{1}{x-\xi}-\frac{\xi}{x \xi-R^{2}}-\frac{\left(\xi^{2}-R^{2}\right) R^{2}}{\xi\left(x \xi-R^{2}\right)^{2}}\left[1-\frac{\xi^{2}-R^{2}}{\xi x-R^{2}}\right]
\end{aligned}
$$

where $\mu$ is the shear modulus, $\kappa=3-4 \nu$ for plane strain and $\nu$ is the Poisson's ratio. It should be noted that the kernels given by $(5,6)$ have been modified in order to accomodate cracks terminating at the hole with opening or shift. The terms dropped correspond to a Volterra dislocation in the infinite medium with the hole. In case of cracks terminating at the hole with stick, the same terms can be dropped by enforcing the auxiliary conditions of uniqueness of displacements:

$$
\int_{b}^{c} B_{y}(\xi) \mathrm{d} \xi=0, \quad \int_{a}^{c} B_{x}(\xi) \mathrm{d} \xi=0 .
$$

Denoting the total normal and shear tractions on $y=0$ by $N(x), S(x)$ we have

$$
\begin{aligned}
& N(x)=\sigma_{y y}(x)+\tau_{y y}(x), \\
& S(x)=\sigma_{x y}(x)+\tau_{x y}(x) .
\end{aligned}
$$

The boundary conditions are now written as follows

$$
\begin{aligned}
& N(x)=0, \quad b<x<c \\
& N(x)<0, \quad a<x<b \\
& S(x)=-f \operatorname{sgn} h(x) N(x), \quad a<x<c \\
& |S(x)|<f|N(x)|, \quad R<x<a
\end{aligned}
$$

where $h(x)$ denotes the total tangential shift related to $B_{x}$ by

$$
B_{x}(x)=-\mathrm{d} h(x) / \mathrm{d} x,
$$

and $f$ is the coefficient of friction. Equations (1), (3), (7a), (8), (10) and (11) permit the determination of the normal tractions and separation related quantities $B_{y}, b$ independently. Using the results obtained thus we solve for the remaining unknowns associated with shear tractions and slip. Each part of the problem reduces to a Cauchy singular integral equation with an auxialiary condition. For the numerical solution we employ the method of Erdogan et al. [8]. The inequalities of the problem are verified a posteriori. Numerical details of similar computations can be found in [9] and [10] and they are omitted here. It suffices to mention that square root singular solutions only occur in transitions from slip or separation to bond [11]. The stress intensity factors are obtained using the interpolation formulas given by Krenk, [12].

In the case of applied shear, Fig. 3, the tractions without the crack are

$$
\begin{aligned}
& \sigma_{y y}(r, \theta)=-T\left(1+\frac{3 R^{4}}{r^{4}}\right) \sin 2 \theta, \\
& \sigma_{x y}(r, \theta)=T\left(1+\frac{2 R^{2}}{r^{2}}-\frac{3 R^{4}}{r^{4}}\right) \cos 2 \theta .
\end{aligned}
$$

The crack remains completely closed for angles $\theta$ in the first and third quadrant and $B_{y}$ vanishes. 


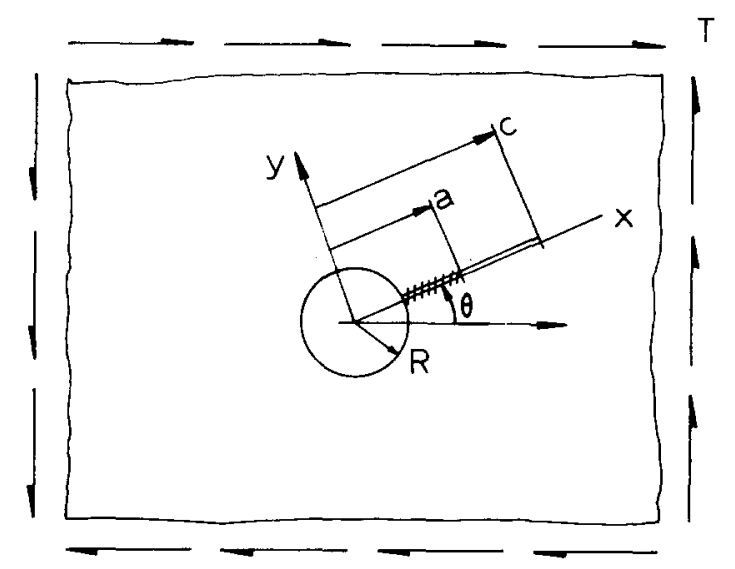

Figure 3. Geometry of the radial crack in an inclined shear field.

\section{Results: tensile load}

When the crack is aligned with the direction of the applied tension, $\theta=0$, the crack is partly open and the contact zone, which starts from the hole, is under conditions of stick. Short cracks, $c<1.74 R$, remain completely closed and ineffective. The dimensionless stress intensity factor $K_{I} / T \sqrt{\delta}$, where $\delta=(c-b) / 2$, is given for various crack lengths in Table 1 . The quantity $K_{I} / T \sqrt{(c-R)}$ is also compared with the corresponding results of [3].

It is noted from Table 1 that for cracks with $c<1.8 R$ the stress intensity factors given in [3] are negative and increasing in magnitude.

As $\theta$ is increased, for cracks that are not too short to have an open region, a zone of slip appears adjacent to the separation and quickly reaches the boundary of the hole. Similarly, the zone of separation extends at the expense of the slip zone until the crack is completely open. The results of Hsu [3] are then recovered. The parameters determining stick, slip and separation and the normalized stress intensity factors in mode I and II for $c=4 R$ and $f=0.5$ are given in Table 2 , where $\Delta=(c-a) / 2$.

The dotted line in Table 2 marks the transition to the case where slip has reached the boundary of the hole, $\theta=4.8^{\circ}$. At angles greater than $19.7^{\circ}$ the crack is fully open. To see the effect of closure and friction we consider the case $\theta=15^{\circ}$, for which results are recorded in [3]. The effect of $c$ on the slip and separation zones and on the stress intensity factors, which are normalized using the effective rather than the original crack length is shown in Table 3 for two values of the coefficient of friction, 0.5 and 0.1 . As noted in the formulation only $a$ and $K_{\text {II }}$ depend on friction. Short cracks tend to stick next to the hole (transition to slip and separation only marked by a dotted line), and there is a critical length $c=1.43 R$ below which cracks remain completely closed. The mode II stress intensity factor increased in magnitude as $f$ decreased. A comparison of the stress intensity factors (normalized using the original crack length) with those of [3] is given in Table 4. The values agree for completely open cracks, $c>7 R$. The algebraic sign of our $K_{\mathrm{II}}$ is opposite of [3]'s because the angles of the load are of opposite signs.

Table 4 shows that the biggest discrepancy in the stress intensity factors occurs when the contact zone is the largest, as expected. For smaller coefficients of friction the discrepancy in $K_{\mathrm{II}}$ is smaller. When the crack is completely open the results agree.

Results for an intermediate angle, $\theta=10^{\circ}$, and $f=0.5$ are given in Table 5. Cracks with $c>2 R$ slip to the boundary of the hole and cracks with $c<1.57 R$ (approximate values) close completely. 
Table 1. $\theta=0^{\circ}, T=$ tension

\begin{tabular}{clllc}
\hline$c / R$ & $b / R$ & $K_{I} / T \sqrt{\delta}$ & $K_{I} / T \sqrt{(c-R)}$ & $K_{I} / T \sqrt{(c-R)},[3]$ \\
\hline 1.1 & 1.1 & 0 & 0 & -0.793 \\
$\ldots$ & $\ldots$ & $\ldots$ & $\ldots$ & $\ldots$ \\
1.8 & 1.711 & 0.008 & 0.002 & -0.123 \\
2 & 1.660 & 0.023 & 0.010 & -0.081 \\
3 & 1.530 & 0.037 & 0.022 & -0.011 \\
4 & 1.470 & 0.030 & 0.020 & 0.002 \\
5 & 1.443 & 0.024 & 0.016 & 0.005 \\
6 & 1.423 & 0.020 & 0.013 & 0.005 \\
7 & 1.410 & 0.016 & 0.011 & 0.005 \\
9 & 1.392 & 0.012 & 0.008 & 0.004 \\
11 & 1.380 & 0.009 & 0.006 & 0.003 \\
21 & 1.359 & 0.004 & 0.003 & 0.002 \\
\hline
\end{tabular}

Table 2. $c / R=4, f=0.5, T=$ tension

\begin{tabular}{lllll}
\hline$\theta^{\circ}$ & $a / R$ & $b / R$ & $K_{1} / T \sqrt{\delta}$ & $K_{11} / T \sqrt{\Delta}$ \\
\hline 1 & 1.270 & 1.470 & 0.031 & -0.021 \\
3 & 1.090 & 1.453 & 0.033 & -0.064 \\
4 & 1.031 & 1.438 & 0.036 & -0.086 \\
4.7 & 1.001 & 1.425 & 0.037 & -0.102 \\
\hline 4.8 & 1.000 & 1.423 & 0.038 & -0.105 \\
6 & & 1.398 & 0.042 & -0.136 \\
7 & 1.374 & 0.046 & -0.163 \\
8 & 1.348 & 0.050 & -0.189 \\
9 & 1.320 & 0.056 & -0.215 \\
10 & & 1.292 & 0.061 & -0.240 \\
11 & & 1.262 & 0.068 & -0.266 \\
12 & 1.232 & 0.075 & -0.292 \\
13 & & 1.202 & 0.083 & -0.316 \\
14 & & 1.171 & 0.091 & -0.341 \\
15 & & 1.140 & 0.100 & -0.366 \\
16 & & 0.110 & -0.389 \\
17 & & 0.120 & -0.413 \\
18 & & 0.108 & 0.143 & -0.435 \\
19 & & 1.076 & 0.150 & -0.457 \\
19.57 & & 1.045 & & -0.469 \\
\hline
\end{tabular}

Table 3. $\theta=15^{\circ}, T=$ tension

\begin{tabular}{|c|c|c|c|c|c|c|}
\hline \multirow[t]{2}{*}{$c / R$} & \multirow[t]{2}{*}{$b / R$} & \multirow[t]{2}{*}{$K_{\mathrm{I}} / T \sqrt{\delta}$} & \multicolumn{2}{|c|}{$f=0.5$} & \multicolumn{2}{|c|}{$f=0.1$} \\
\hline & & & $a / R$ & $K_{\mathrm{II}} / T \sqrt{\Delta}$ & $a / R$ & $K_{\mathrm{II}} / T \sqrt{\Delta}$ \\
\hline 1.43 & 1.428 & 0.001 & 1.030 & -0.253 & \multirow[t]{10}{*}{1.000} & -0.339 \\
\hline 1.5 & 1.406 & 0.022 & 1.010 & -0.279 & & -0.361 \\
\hline$\overline{1.6}$ & 1.379 & 0.046 & 1.000 & -0.312 & & -0.384 \\
\hline 1.8 & 1.335 & 0.076 & & -0.358 & & -0.407 \\
\hline 2 & 1.301 & 0.092 & & -0.380 & & -0.416 \\
\hline 3 & 1.196 & 0.106 & & -0.386 & & -0.398 \\
\hline 4 & 1.140 & 0.100 & & -0.366 & & -0.370 \\
\hline 5 & 1.102 & 0.094 & & -0.348 & & -0.350 \\
\hline 6 & 1.073 & 0.089 & & -0.334 & & -0.335 \\
\hline 7 & 1.051 & 0.086 & & -0.323 & & -0.324 \\
\hline
\end{tabular}


Table 4. $\theta=15^{\circ}, f=0.5, T=$ tension

\begin{tabular}{lllll}
\hline$c / R$ & $K_{\mathrm{I}} / T \sqrt{(c-R)}$ & $K_{\mathrm{I}} / T \sqrt{(c-R)},[3]$ & $\left|K_{\mathrm{II}} / T \sqrt{(c-R)}\right|$ & $K_{\mathrm{II}} / T \sqrt{(c-R)},[3]$ \\
\hline 1.5 & 0.007 & -0.115 & 0.197 & 0.271 \\
1.6 & 0.020 & -0.073 & 0.221 & 0.284 \\
1.8 & 0.041 & -0.019 & 0.253 & 0.296 \\
2 & 0.054 & 0.048 & 0.269 & 0.300 \\
3 & 0.071 & 0.059 & 0.273 & 0.283 \\
4 & 0.069 & 0.064 & 0.259 & 0.263 \\
5 & 0.066 & 0.063 & 0.246 & 0.248 \\
6 & 0.063 & 0.061 & 0.236 & 0.237 \\
7 & 0.060 & 0.060 & 0.229 & 0.229 \\
\hline
\end{tabular}

Table 5. $\theta=10^{\circ}, f=0.5, T=$ tension

\begin{tabular}{lcccc}
\hline$c / R$ & $b / R$ & $a / R$ & $K_{\mathrm{I}} / T \sqrt{\delta}$ & $K_{\mathrm{II}} / T \sqrt{\Delta}$ \\
\hline 1.2 & & 1.190 & -0.007 \\
1.3 & 1.159 & & -0.078 \\
1.4 & 1.133 & -0.129 \\
1.5 & 1.106 & -0.163 \\
-1.57 & & 1.089 & -0.183 \\
1.7 & 1.060 & 0.001 & -0.203 \\
1.8 & 1.527 & 1.040 & 0.024 & -0.212 \\
2 & 1.503 & 1.007 & 0.037 & -0.223 \\
-3 & 1.462 & 0.052 & -0.248 \\
4 & 1.348 & & -0.067 & -0.231 \\
5 & 1.292 & & 0.061 & -0.224 \\
6 & 1.256 & & 0.055 & -0.217 \\
7 & 1.230 & & 0.051 & -0.209 \\
9 & 1.211 & & 0.047 & -0.202 \\
11 & 1.181 & & 0.043 & -0.188 \\
21 & 1.159 & & 0.040 & 0.034 \\
\hline
\end{tabular}

\section{Results: shearing load}

The geometry for the shearing load is shown in Fig. 3. The crack remains completely closed in the first and third quadrant. Because of the symmetries involved we need to examine only the range $0<\theta<\pi / 4$ for the closed crack. For $\theta=0$ the normal tractions vanish and the results must be the same as in the frictionless case. A comparison with [3] is given in Table 6. As $\theta$ is increased slip occurs in the positive direction along the entire length of the crack. At some angle less than $\pi / 4$ a zone of stick appears next to the hole boundary and eventually covers the entire crack extent. Slip in the opposite direction occurs at the angle symmetric with respect to $\pi / 4$. The results are summarized in Table 7 for $c=3.5 R$, and $f=0.5$ and $f=0$. In the latter case stick only occurs at $\theta=\pi / 4$. Friction reduces the stress intensity factor as expected. The dotted line marks the start of complete stick along the crack and $\Delta=(c-a) / 2$ as in the previous section. Note also that the stress intensity factors are bigger for $\theta=0$, a fact that is obscured by the different normalization used in Table 6 for comparison with [3].

For reference purposes we have also recorded stress intensity factors for the completely open crack. $\pi / 2<\theta<3 \pi / 4$ and $c=2 R$ in Table 8 , where $\delta=(c-b) / 2$ as in the previous section. 
Table 6. $\theta=0^{\circ}, T=$ shear

\begin{tabular}{lll}
\hline$c / R$ & $K_{\mathrm{II}} / T \sqrt{(c-R)}$ & $K_{\mathrm{II}} / T \sqrt{(c-R)},[3]$ \\
\hline 1.1 & 0.436 & 0.420 \\
1.2 & 0.712 & 0.706 \\
1.3 & 0.891 & 0.886 \\
1.4 & 1.008 & 1.008 \\
1.5 & 1.085 & 1.086 \\
1.6 & 1.135 & 1.134 \\
1.8 & 1.186 & 1.186 \\
2 & 1.201 & 1.200 \\
2.5 & 1.177 & 1.176 \\
3 & 1.132 & 1.132 \\
4 & 1.051 & 1.050 \\
5 & 0.991 & $0.922 *$ \\
6 & 0.948 & 0.948 \\
7 & 0.916 & 0.916 \\
9 & 0.872 & 0.872 \\
21 & 0.779 & 0.770 \\
$\infty$ & 0.707 & 0.707 \\
\hline
\end{tabular}

* This value appears to have been misprinted.

Table 7. $c / R=3.5, T=$ shear

\begin{tabular}{cllll}
\hline $\begin{array}{l}f=0.5 \\
\theta^{\circ}\end{array}$ & $a / R$ & $K_{\mathrm{II}} / T \sqrt{\Delta}$ & $f=0$ & $K_{\mathrm{II}} / T \sqrt{\Delta}$ \\
\hline 0 & 1.000 & 1.540 & 1.000 & 1.540 \\
10 & 1.000 & 1.168 & & 1.447 \\
20 & 1.000 & 0.655 & & 1.180 \\
30 & 1.288 & 0.139 & 0.769 \\
33 & 1.910 & 0.005 & 0.626 \\
$-\frac{-13.3}{40}$ & & & 0.612 \\
45 & & & 0.267 \\
\hline
\end{tabular}

Table 8. $c / R=2, b / R=1, a / R=1, T=$ shear

\begin{tabular}{rlc}
\hline$\theta^{\circ}$ & $K_{\mathrm{I}} / T \sqrt{\delta}$ & $K_{\mathrm{II}} / T \sqrt{\Delta}$ \\
\hline 91 & 0.068 & -1.697 \\
100 & 0.670 & -1.595 \\
110 & 1.260 & -1.301 \\
120 & 1.698 & -0.845 \\
130 & 1.931 & -0.295 \\
135 & 1.960 & 0 \\
\hline
\end{tabular}

\section{Acknowledgement}

The first author acknowledges the support of the Office of Naval Research through contract N00014-84-k-0287 during the course of this research. Work along similar lines was originally planned in collaboration with the late Professor Y.C. Hsu. This paper is dedicated to his memory. 


\section{References}

[1] O.O. Bowie, Journal of Mathematics and Physics 35 (1956) 60-71.

[2] J. Tweed and D.P. Rooke, International Journal of Engineering Science 11 (1973) 1185-1195.

[3] Y.C. Hsu, International Journal of Fracture 11 (1975) 571-581.

[4] C. Atkinson, International Journal of Engineering Science 10 (1972) 127-136.

[5] F. Erdogan and G.D. Gupta, International Journal of Fracture 11 (1975) 11-27.

[6] S.P. Timoshenko and J.N. Goodier, Theory of Elasticity, McGraw-Hill (1970).

[7] J. Dundurs and T. Mura, Journal of Mechanics and Physics of Solids 12 (1964) 177-189.

[8] F. Erdogan, G.D. Gupta and T.S. Cook, in Methods of Analysis and Solution of Crack Problems. Edited by G.C. Sih. Noordhoff International Publishing (1973).

[9] M. Comninou, D. Schmueser and J. Dundurs, International Journal of Engineering Science 17 (1980) 131-137.

[10] D.A. Hills and M. Comninou, International Journal of Solids and Structures, in press.

[11] J. Dundurs and M. Comninou, Journal of Elasticity 9 (1979) 71-82.

[12] S. Krenk, Quarterly of Applied Mathematics 32 (1975) 479-484.

\section{Résumé}

On considère une fissure radiale provenant d'un trou circulaire dans une tôle mince infinie sous tension. Selon la longueur et la localisation de la fissure, celle-ci peut être partiellement ou totalement ouverte ou fermeée. Dans ce dernier cas, la résolution du champ de contrainte est influencée par la friction entre faces de la fissure.

On calcule les facteurs d'intensité de contrainte correspondant à une tension dont l'axe est incliné par rapport à la fissure, ou à un cisaillement dans le plan, et on compare les valeurs obtenues à celles correspondant aux cas où l'on ignore la fermeture de la fissure et la friction entre ses faces. 